\title{
Study of the modulation capabilities of parallel aligned liquid crystal on silicon displays
}

\author{
A. Márquez ${ }^{1,2, *}$, F. J. Martínez ${ }^{1,2}$, S. Gallego ${ }^{1,2}$, M. Ortuño ${ }^{1,2}$, J. Francés ${ }^{1,2}$, A. Beléndez ${ }^{1,2}$, I. \\ Pascual $^{2,3}$ \\ ${ }^{1}$ Dept. de Física, Ing. de Sistemas y T. Señal, Univ. de Alicante, Ap. 99, E-03080, Alicante, Spain \\ ${ }^{2}$ I.U. Física Aplicada a las Ciencias y las Tecnologías U. de Alicante, Ap. 99, E-03080, Alicante, Spain \\ ${ }^{3}$ Dept. de Óptica, Farmacología y Anatomía, Univ. de Alicante, Ap. 99, E-03080, Alicante, Spain
}

\begin{abstract}
Among the existent technologies of spatial light modulator devices, parallel aligned liquid crystal on silicon displays (PA-LCoS) have found wide acceptance. They are especially interesting since they provide phase-only modulation with no coupling of amplitude modulation. Optimal use of these devices requires proper calibration of their modulation capabilities in order to minimize some degradation effects found in the literature, such as flicker or anamorphic and frequency dependent modulation. In this work we apply calibration techniques developed in our lab, basically the classical linear polarimeter adapted to be able to take into account the existence of flicker. This method enables to obtain both the average retardance and a good estimation of the magnitude of the phase fluctuation when flicker is present. Various addressing formats are discussed and variations in the magnitude of the applied voltages are considered in order to amplify the retardance dynamic range and to enhance linearity in the device. Finally, two electrical configurations intended for phase-only and amplitude-mostly modulation regimes, useful e.g. in diffractive optics, are given.
\end{abstract}

Keywords: Liquid crystal on silicon displays, parallel aligned, retardance measurement, phase-only modulation, spatial light modulation, flicker, diffractive optics.

\section{INTRODUCTION}

Liquid crystal (LC) microdisplays have become a central device in a wide range of applications requiring spatial modulation of a wavefront like in diffractive optics ${ }^{[1]}$, optical storage ${ }^{[2]}$, or optical metrology ${ }^{[3]}$. Liquid crystal on silicon (LCoS) displays have become the most attractive microdisplays for these applications due to their very high spatial resolution and very high light efficiency ${ }^{[4][5]}$. However, several authors ${ }^{[6]-[10]}$ have detected that LCoS displays produce certain amount of phase flicker and/or depolarization. Other degradation effects exist, such as the anamorphic and frequency dependent modulation, which was originally detected in $\operatorname{LCDs}^{[11][12]}$, and also reported in both digital ${ }^{[13]}$ and analog ${ }^{[14]}$ LCoS devices. Among the different LCoS technologies typically available, parallel aligned LCoS (PA-LCoS) are especially interesting since they allow easy operation as phase-only devices without coupled amplitude modulation. In this case, no depolarization operation is readily available, however phase fluctuations are still a feature in digitally addressed LCoS devices ${ }^{[15]}$.

Different measurement methods have been proposed and demonstrated to characterize the phase-shift versus voltage in LCoS devices ${ }^{[16][17]}$. Some of these methods provide full polarimetric characterization and are based on Mueller-Stokes polarimeters. Other methods are especifically oriented to obtain phase-shift modulation values such as the diffractive and the interferometric methods described by Lizana et al. ${ }^{[16][17]}$ : the first method is very well suited for instantaneous values measurement, whereas the latter is appropriate to obtain the average values. These methods can be applied to LCDs and LCoS devices in general. In the case of parallel aligned devices, they are totally characterized by their linear retardance vs. voltage values. Additional methods typically used in the characterization of waveplates become available to measure the linear retardance as discussed in Ref. [18] and [19]. One common property to all these methods is that they assume that the birefringence in the wave plate has a constant value, no fluctuations, during the measurement process.

In Ref. [19] we analyzed how the classical method used to measure the linear retardance of waveplates can be extended to characterize the retardance in the presence of fluctuations or instabilities. We demonstrated that the classical approach produces wrong results, however with an adequate modeling of the experiment we showed that the classical approach

Optics and Photonics for Information Processing VII, edited by Khan M. Iftekharuddin,

Abdul A. S. Awwal, Andrés Márquez, Proc. of SPIE Vol. 8855, 885504 · C 2013 SPIE

CCC code: $0277-786 X / 13 / \$ 18 \cdot$ doi: $10.1117 / 12.2023266$ 
can be extended not only to obtain correct values of the retardance but also a good estimation of the flicker amplitude at specific retardance points. In some other paper ${ }^{[20]}$ Ramírez et al. showed that with some additional measurements, involving not only the linear but also the circular polarimeter, both the retardance and the visibility contrast (related with the flicker amplitude) may be in general obtained, and not only at specific retardance values. These strategies are useful to provide accurate calibration of electrooptic devices which can be assimilated to linear retarders. This is for example the case of the PAL-LCoS in Ref. [19] and the liquid crystal cell in Ref. [20].

In the present work we take advantage of the ease of implementation and the effectiveness of the calibration method proposed in Ref. [19] to inspect the configuration options given for a specific LCoS model and their effect on the average linear retardance dynamic range and the magnitude of the flicker. This enables to find electrical configurations which may be used for phase-only or for amplitude-mostly modulation regimes. In Section 2 we describe the calibration method. In Section 3 we show the configuration options available for a certain LCoS model. These options, which are quite generic, offer the user the capability to obtain a fine adjustment of the modulation regime of the LCoS, adapted for the specific application. In Section 4 we give the experimental results and, eventually, in Section 5 the main conclusions are presented.

\section{CHARACTERIZATION WITH A LINEAR POLARIMETER}

In the following we introduce the extended linear polarimeter proposed in Ref. [19], which provides a calibration that can be enough in most of the situations. In Fig. 1 we show the basic scheme of a linear polarimeter, where P1 and P2 correspond to the input and output polarizers, and WP corresponds to the waveplate whose linear retardance $\Gamma$ needs to be measured. The angles $\theta_{1}$ and $\theta_{2}$ correspond respectively to the orientation of the transmission axis with respect to the vertical lab axis (X-axis) and $\Omega$ is analogously the angle of the neutral lines (in the figure the slow axis) for the waveplate. In the analysis showed in Ref. [18] it was demonstrated that the classical technique based on measuring the intensity with a radiometer at the exit of the system for the parallel and crossed configurations of the polarizers, both oriented at $45^{\circ}$ or $-45^{\circ}$ with respect to the neutral lines of the waveplates, is not only a simple method but also the most robust to misalignments and other types of noise sources in the system.
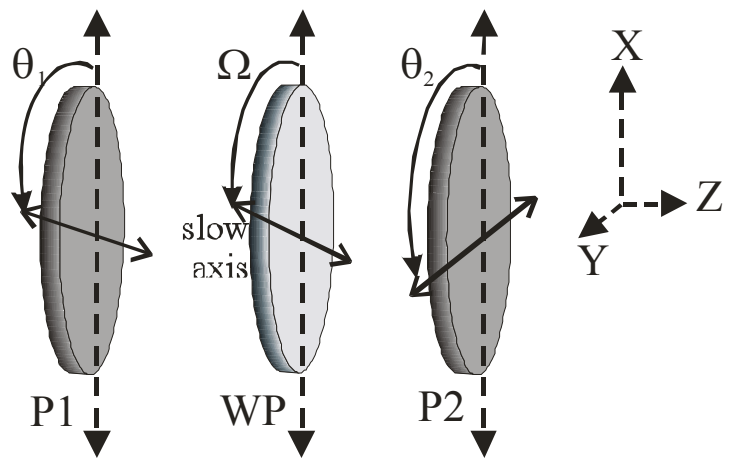

Figure 1. Linear polarimeter with the wave plate WP to be measured. P1 and P2 are the polarizers.

In the case of instabilities or fluctuations in the linear retardance, as it is the case in digitally addressed LCoS device ${ }^{[21][22]}$ and in other electrooptic devices ${ }^{[20]}$, as a first approximation we may consider a triangular profile for the periodic variation of retardance with time $\Gamma(t)$, with an average value $\bar{\Gamma}$, retardance fluctuation amplitude $a$, and period $T$, i.e. described by the following equation,

$$
\Gamma(t)= \begin{cases}\bar{\Gamma}-a+\frac{2 a}{T / 2} t & 0 \leq t<T / 2 \\ \bar{\Gamma}+3 a-\frac{2 a}{T / 2} t & T / 2 \leq t<T\end{cases}
$$

In Ref. [19] we showed that when applying this temporal fluctuation profile the averaged parallel and crossed polarizers intensities become, 


$$
\begin{aligned}
& \left\langle I_{\text {OUT }}^{\prime \prime}\right\rangle=\frac{I_{0}}{2}\left[1+\frac{\sin a}{a} \cos \bar{\Gamma}\right] \\
& \left\langle I_{\text {OUT }}^{\perp}\right\rangle=\frac{I_{0}}{2}\left[1-\frac{\sin a}{a} \cos \bar{\Gamma}\right]
\end{aligned}
$$

And when combining the two expressions we obtain,

$$
\frac{\left\langle I_{\text {OUT }}^{\prime \prime}\right\rangle-\left\langle I_{\text {OUT }}^{\perp}\right\rangle}{\left\langle I_{\text {OUT }}^{\prime \prime}\right\rangle+\left\langle I_{\text {OUT }}^{\perp}\right\rangle}=\frac{\sin a}{a} \cos \bar{\Gamma}
$$

, where we see that the cosine term becomes modulated by the presence of a sinc function dependent on the magnitude of the fluctuation $a$. This sinc function represents the deviation from the classical expression typically used to obtain the linear retardance value.

The maxima and minima in the parallel and crossed polarizers intensity curves are produced at average values $\bar{\Gamma}$ which are multiple of $180^{\circ}$. At these points when there is no flicker we obtain that the maxima and minima of the normalized intensity are equal to 1 and 0 . When flicker is present the maxima and minima values deviate from 1 and 0 . The absolute value of this difference in intensity, $I_{\text {diff }}$, is equal to,

$$
I_{\text {diff }}=0.5\left(1-\frac{\sin a}{a}\right)
$$

In Figure 2(a) we represent $I_{\text {diff }}$ versus the magnitude of the fluctuation $a$, calculated using Eq. (5). We see the nonlinear increase of $I_{\text {diff }}$ with the increase of the fluctuation due to the sinc function. If this plot is reversed, Figure 2(b), we have a straightforward way to estimate the magnitude of the fluctuation simply by looking at the deviation in the intensity at the maxima or minima in the intensity, i.e. by selecting the points where the average value $\bar{\Gamma}$ is multiple of $180^{\circ}$. On the figure we show (dashed line) the polynomial fit to the curve, using a $6^{\text {th }}$ degree polynomial function. For the sake of completeness we show the squared correlation factor, which is very close to one. Using this polynomial we can quantitatively obtain the magnitude of the fluctuation from the value of $I_{\text {diff }}$.
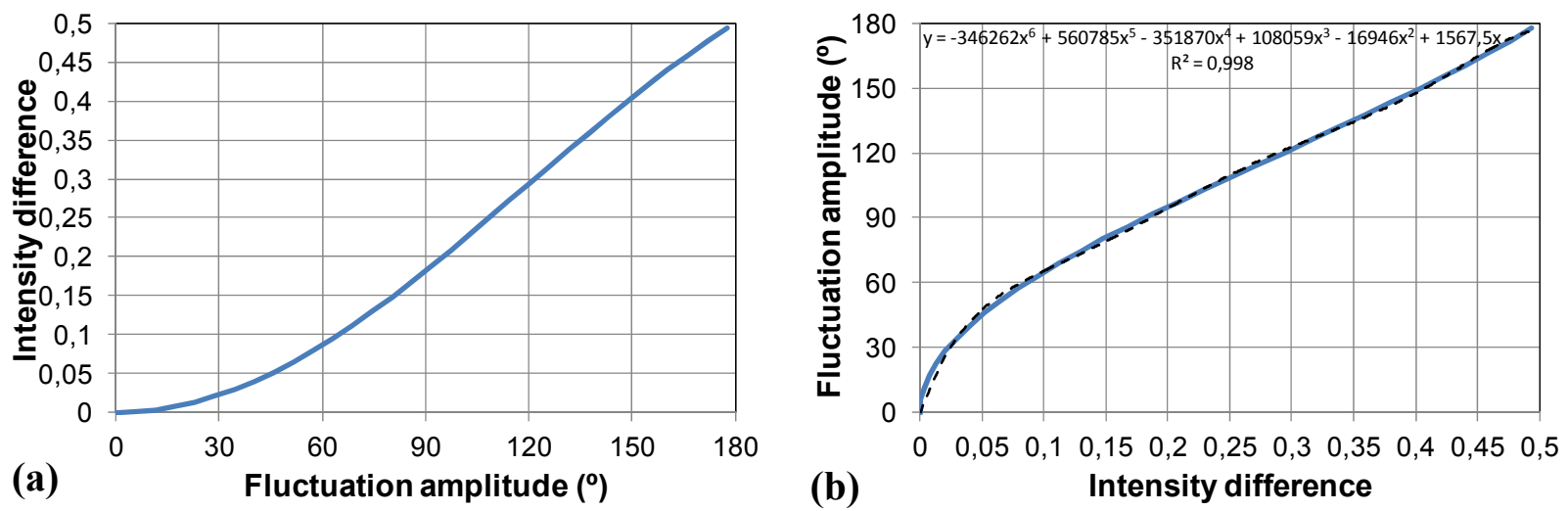

Figure 2. (a) Intensity difference (absolute value) with respect to the ideal $a=0^{\circ}$ as a function of the fluctuation amplitude, at the maxima and minima intensity points, occurring when the average value $\bar{\Gamma}$ is multiple of $180^{\circ}$; (b) Reverse relation, where a polynomial fit has been calculated with respect to the theoretical curve. 
In Figure 3 we present the experimental setup that we use to obtain the parallel and crossed polarizers intensities. It consists of a linear polarimeter, where the reflective LCoS is the linear retarder to be measured. The setup is intended both to obtain average values and instantaneous intensity measurements. The latter is straightforward due to the nonpolarizing beam-splitter before the analyzers which allows for synchronized parallel and crossed polarizers intensity measurement. A quarter wavelength waveplate is added after the laser so that enough light passes through the first polarizer irrespectively of the orientation of its transmission axis. Note that in the geometry in Fig. 3, actually the LCoS is characterized in combination with the beam-splitter situated in front. Obviously, other working geometries for the $\mathrm{LCoS}$ are also possible, i.e. $45^{\circ}$ incidence angle, and may require appropriate characterization.

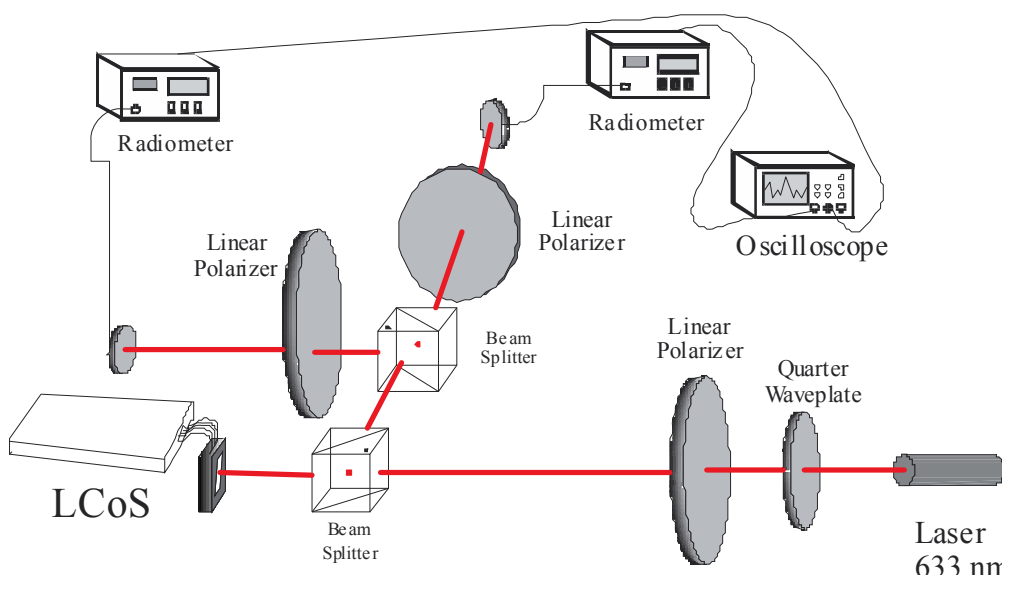

Figure 3. Experimental setup used to measure the linear retardance as a function of the applied voltage (gray level) for a PALCoS. The setup allows both to measure average and instantaneous values.

\section{LCOS CONFIGURATION OPTIONS AND MODULATION RANGE ADAPTION}

In the paper we analyze a digitally addressed phase-only LCoS. It corresponds to an electrically controlled birefringence (ECB) LCoS display distributed by the company HOLOEYE. It is an active matrix reflective mode device with 1920x1080 pixels and 0.7" diagonal named the PLUTO Spatial Light Modulator (SLM). The pixel pitch is of $8.0 \mu \mathrm{m}$ and the display has a fill factor of $87 \%$. The signal is addressed via a standard DVI (Digital Visual Interface) signal. By means of the RS-232 interface and its corresponding provided software, we can configure a series of interesting electrical parameters of the device ${ }^{[21][22]}$ such as the digital addressing sequence format (bit-planes), the gamma curve, and the voltage dynamic range of the pulse width modulated (PWM) signal (through two digital potentiometers).

In particular the software for the SLM is provided with a series of configuration files corresponding to two different digital addressing sequences: the so-called 18-6 and 5-5 digital sequences. The first number indicates the quantity of "equally weighted" bit-planes, and the second number the quantity of "binary" bit-planes ${ }^{[21]}$. This means that the sequence 18-6 is longer than the one corresponding for the sequence 5-5. In principle the shorter the sequence the smaller the flicker ${ }^{[21]}$. However, a larger sequence provides a larger number of possible phase levels: $(18+1) \times 2^{6}=1216$ for the sequence " $18-6$ " and $(5+1)$ × $2^{5}=192$ for the sequence "5-5". For each of the sequences we find a series of configuration files which have been in principle optimized by the vendor for different wavelengths to enable non-expert SLM users a fast access to a proper operating configuration for the device adapted to the specific application and illuminating wavelength. In general, these configuration files enable phase-only operation with a $2 \pi$ linear phase dynamic range for the specified wavelength. To this goal the digital potentiometer values and the gamma curve, also called look-up table, LUT, need to be properly selected.

Applying the technique presented in Section 2 we have a fast and direct way to discriminate, in terms of the average retardance dynamic range and the magnitude of the flicker, between the digital addressing sequences provided with the software and driver electronics. Finer adjustment of the retardance dynamic range and its linearity is then feasible by modifying the gamma curve and changing the digital potentiometers values. The simplicity of the linear polarimeter 
setup on which the method is based allows non-expert SLM users to have access to adapt the calibration to their specific working geometry and illuminating wavelength without the need to rely on the finite series of configuration files provided by the vendor.

In the following we restrict our attention to sequence 5-5 since it produces less flicker than sequence 18-6, as previously commented and as already demonstrated in a number of papers ${ }^{[17][19][21]}$. In Fig. 4(a), (b) and (c) we show three examples of gamma curves, as given by the LCoS software interface, for the 5-5 sequence, corresponding respectively to the configuration files named "5-5 default ramp", "5-5 linear 2pi 633nm" and "5-5 linear 2pi 405nm". As we will see in Section 4, the first configuration provides the largest voltage modulation range available, so that we can estimate the maximum retardance dynamic range provided by the LCoS unit. The second and third configurations span a retardance range of $2 \pi$ and $\pi$ radians respectively, which are the necessary ranges to produce respectively a phase-only regime and an amplitude-mostly regime when using a variable linear retarder.

In Fig. 4(a) we see that there is a linear mapping between the input and output values. We also see that all the 192 possible values associated with the 5-5 sequence are available. In Fig. 4(b) the mapping is highly nonlinear, especially for lower gray levels, and only 95 out of the 192 possible values are available. In Fig. 4(c) the mapping is rather linear and a large quantity of gray levels, 175 , are available.
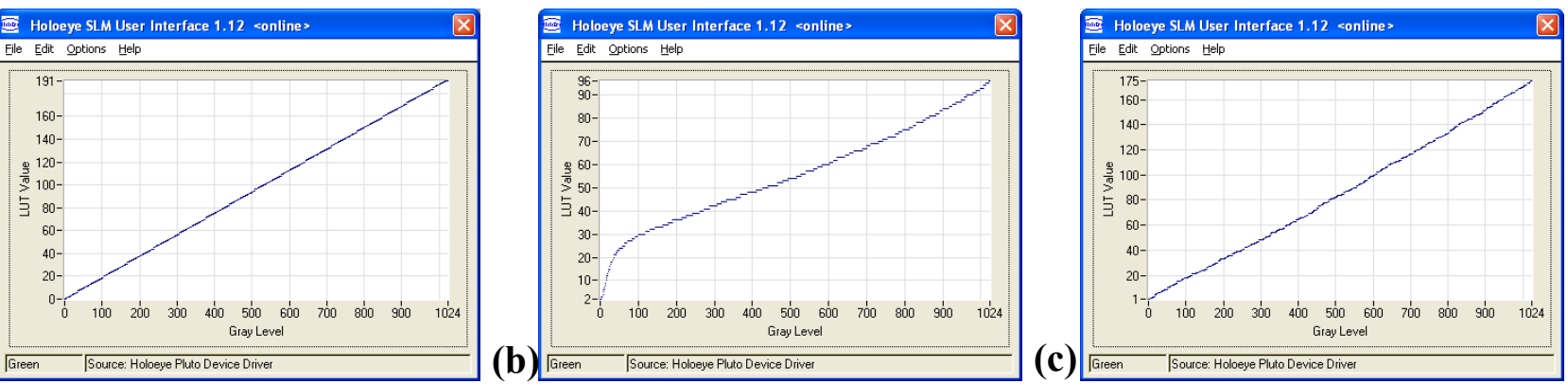

Figure 4. Gamma curves as shown by the PLUTO LCoS software interface for the configuration files: (a) "5-5 default ramp", (b) "5-5 linear 2pi 633nm", and "5-5 linear 2pi 405nm".

In Fig. 5(a) and (b) we show the values for the digital potentiometers, as given by the LCoS software interface: (a) corresponds both to the configuration file " $5-5$ default ramp" and " $5-5$ linear 2 pi $633 \mathrm{~nm}$ ", and (b) corresponds to the configuration " $5-5$ linear 2pi $405 \mathrm{~nm}$ ". We see that the values for the two digital resistors are associated with two voltage values, $V_{\text {bright }}$ and $\mathrm{V}_{\text {dark }}$. A change in these values affects the voltage amplitude of the PWM pulses, thus affecting the accumulated voltage experienced by the LC molecules in a whole frame period. We see that in Fig. 5(a) the voltage range is much larger than for Fig. 5(b). Actually, the largest voltage range possible is 3.82-0.03V, given in Fig. 5(a).
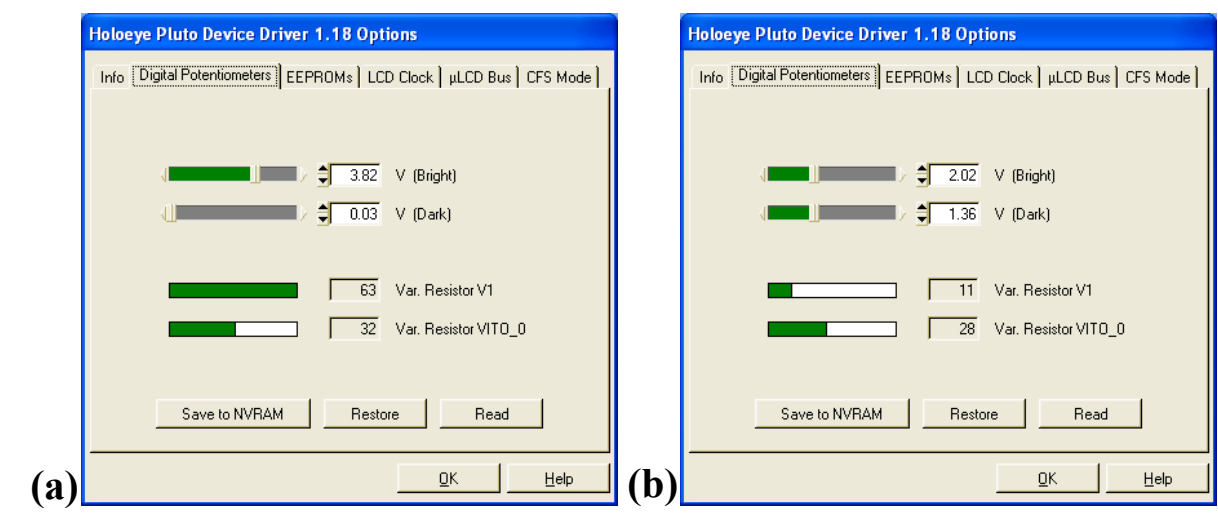

Figure 5. Values for the digital potentiometers, as given by the LCoS software interface: (a) corresponds both to the configuration file "5-5 default ramp" and "5-5 linear 2pi 633nm", and (b) corresponds to the configuration "5-5 linear 2pi $405 \mathrm{~nm}$ ". The values for the two digital resistors are associated with two voltage values, $\mathrm{V}_{\text {bright }}$ and $\mathrm{V}_{\text {dark. }}$. 


\section{RESULTS AND DISCUSSION}

To measure both the parallel and crossed polarizers intensities we consider the input polarizer transmission axis at $+45^{\circ}$ to the vertical of the lab, which is the $\mathrm{X}$-axis of our reference system as shown in Figure 1. The director for the liquid crystal (i.e. the extraordinary axis) in the LCoS is oriented along the horizontal, i.e. at $+90^{\circ}$ with respect to the $\mathrm{X}$-axis. The director axis in nematic filled devices, as it is the case for our LCoS typically corresponds to the slow axis. Light impinges perpendicularly onto the LCoS and onto the two beam-splitters, see Figure 3. Perpendicular incidence onto the beam-splitters is important so that they do not introduce polarization effects on their own. Typically the angular uncertainty on the orientation of the input and output polarizers with respect to the neutral lines of the LCoS can be considered $\pm 1^{\circ}$.

In Figure 6 we plot, first column, the normalized intensity values measured for the parallel and crossed polarizer configurations versus the applied gray level and, second column, the corresponding retardance (wrapped values) calculated using the classical approach (uncorrected curves) and the extended calibration method (corrected curves). In the first, second and third rows are given respectively the results for the configurations "5-5 default ramp", "5-5 linear 2pi $633 \mathrm{~nm}$ " and "5-5 linear 2pi 405nm". From the intensity difference, $I_{\text {diff }}$, at the extremals in the curves in the first column, and applying the polynomial in Figure 2(b), the fluctuation amplitude $a$ can be calculated. These values are presented in Table 1. We see that the fluctuation amplitude increases with the applied gray level: for example, for the configuration " $5-5$ default ramp" the successive extremals exhibit fluctuation amplitudes of $14^{\circ}, 34^{\circ}$ and $43^{\circ}$. The values in Table 1 are then used to produce the corrected average retardance curves in the second column in Figure 6.

Table 1. Fluctuation amplitude $a$ (third column) calculated for three electrical configuration files (first column), using the values for the intensity difference (second column) at the extremals for the parallel (or crossed) polarizers intensity curves as given by the calibration method in Ref. [19].

\begin{tabular}{lll}
\hline Sequence & $I_{\text {diff }}\left(^{\circ}\right)$ & $a\left(^{\circ}\right)$ \\
\hline \multirow{2}{*}{ 5_5 default ramp } & $0.005(\mathrm{GL}$ 40) & 14 \\
& $0.029(\mathrm{GL} \mathrm{80)}$ & 34 \\
& $0.045(\mathrm{GL} \mathrm{140)}$ & 43 \\
5_5 linear 2pi 633nm & $0.0033(\mathrm{GL} 26)$ & 12 \\
& $0.032(\mathrm{GL} \mathrm{155)}$ & 36 \\
5_5 linear 2pi 405nm & 0.0037 (GL 210) & 12 \\
\hline
\end{tabular}

If we take a look at the plots in Figure 6, we observe that the intensity curves in the first row, configuration " $5-5$ default ramp", present a larger number of oscillations, which indicates a larger retardance dynamic range. If the retardance is unwrapped in Figure 6(a2) we obtain a dynamic range of about $560^{\circ}$, then exceeding the necessary $360^{\circ}$ for phase-only modulation. We observe, Fig. 6(a2), that the uncorrected and corrected retardance curves only deviate at values multiple of $180^{\circ}$. We also see that the retardance presents a nonlinear relation with the gray level. In Figure 6(b2) for the configuration " $5-5$ linear 2pi $633 \mathrm{~nm}$ ", the retardance dynamic range is slightly higher than $360^{\circ}$, and the relation with the gray level is very linear. Therefore the gamma curve and the $V_{\text {bright }}$ and $V_{\text {dark }}$ values in Fig. 4 and 5 have been properly tuned and this configuration may be used for phase-only operation: this requires to incide with linearly polarized light along the molecular director of the LCoS (along the horizontal). In Figure 6(c2) the curve shows a retardance range about $180^{\circ}$. This is appropriate to obtain amplitude-mostly operation with a very good intensity contrast. This is actually what we obtained in Fig. 6(c1) for the crossed polarizers. 

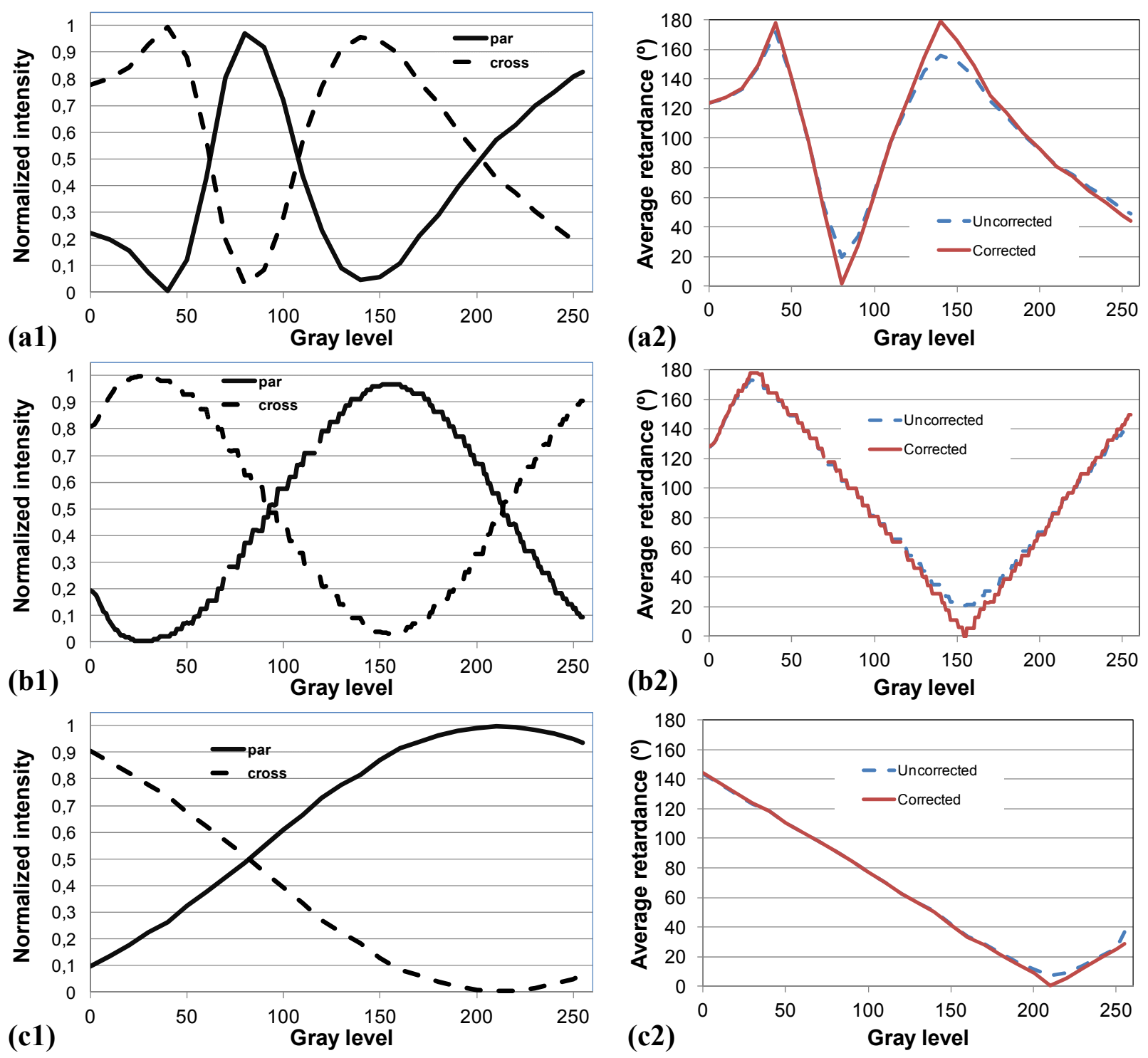

Figure 6. Normalized intensity in (a1), (b1) and (c1) measured for the parallel and crossed polarizer configurations versus the applied gray level, and corresponding retardance (wrapped values) in (a2), (b2) and (c2) calculated using the classical approach (uncorrected curves) and the extended calibration method (corrected curves). In the first, second and third rows are given respectively the results for the configurations "5-5 default ramp", "5-5 linear 2pi 633nm" and "5-5 linear 2pi 405nm".

\section{CONCLUSIONS}

In this work we have applied the extended linear polarimeter method to analyze the various electrical configurations provided with the software for a digitally addressed LCoS device, in terms of the average retardance dynamic range and the magnitude of the flicker. Proper choice of the digital addressing sequence, together with the fine adjustment available via the software options given by the gamma curve and the digital potentiometers controls, have enabled to obtain two electrical configurations intended for phase-only and amplitude-mostly modulation regimes, useful e.g. in diffractive optics. 


\section{ACKNOWLEDGEMENTS}

This work was supported by the Ministerio de Trabajo y Competitividad of Spain under projects FIS2011-29803-C02-01 and FIS2011-29803-C02-02 and by the Generalitat Valenciana of Spain (projects PROMETEO/2011/021 and ISIC/2012/013).

\section{REFERENCES}

[1] J. Turunen and F. Wyrowski Edts., [Diffractive Optics for Industrial and Commercial Applications], Akademie Verlag, Berlin, (1997).

[2] H. J. Coufal, D. Psaltis and B. T. Sincerbox, Eds., [Holographic Data Storage], Springer-Verlag, Berlin, (2000).

[3] W. Osten, C. Kohler and J. Liesener, "Evaluation and application of spatial light modulators for optical metrology," Opt. Pura Apl. 38, 71-81 (2005).

[4] S. T. Wu and D. K. Yang, [Reflective Liquid Crystal Displays], John Wiley \& Sons Inc., Chichester, (2005).

[5] N. Collings, T. Davey, J. Christmas, D. Chu, and B. Crossland, "The Applications and Technology of PhaseOnly Liquid Crystal on Silicon Devices," J. Display Technol. 7, 112-119 (2011).

[6] J. E. Wolfe, and R. A. Chipman, "Polarimetric characterization of liquid-crystal-on-silicon panels," Appl. Opt. 45, 1688-1703 (2006).

[7] A. Márquez, I. Moreno, C. Iemmi, A. Lizana, J. Campos and M. J. Yzuel, "Mueller-Stokes characterization and optimization of a liquid crystal on silicon display showing depolarization," Opt. Express 16, 1669-1685 (2008).

[8] P. Clemente, V. Durán, Ll. Martínez-León, V. Climent, E. Tajahuerce, and J. Lancis, "Use of polar decomposition of Mueller matrices for optimizing the phase response of a liquid-crystal-on-silicon display," Opt. Express 16, 1965-1974 (2008).

[9] J. García-Márquez, V. López, A. González-Vega, and E. Noé, "Flicker minimization in an LCoS spatial light Modulator," Opt. Express 20, 8431-8441 (2012).

[10]A. Lizana, I. Moreno, C. Iemmi, A. Márquez, J. Campos and M. J. Yzuel, "Time-resolved Mueller matrix analysis of a liquid crystal on silicon display," Appl. Opt. 47, 4267-4274 (2008).

[11]A. Márquez, C. Iemmi, I. Moreno, J. Campos and M. J. Yzuel, "Anamorphic and spatial frequency dependent phase modulation on liquid crystal displays. Optimization of the modulation diffraction efficiency," Opt Express 13, 2111-2120 (2005).

[12]A. Márquez, I. Moreno, C. Iemmi, J. Campos and M. J. Yzuel, "Electrical origin and compensation for two sources of degradation of the spatial frequency response exhibited by liquid crystal displays," Opt. Eng. 46, 114001 1-7 (2007).

[13]L. Lobato, A. Lizana, A. Márquez, I. Moreno, C. Iemmi, J. Campos and M. J. Yzuel, "Characterization of the anamorphic and spatial frequency dependent phenomenon in Liquid Crystal on Silicon displays," J. Eur. Opt. Soc. - Rapid Pub. 6, 11012S 1-6 (2011).

[14]J. Albero, P. García-Martínez, J. L. Martínez, and I. Moreno, "Second order diffractive optical elements in a spatial light modulator with large phase dynamic range," Opt. Lasers Eng. 51, 111-115 (2013).

[15]A. Lizana, I. Moreno, A. Márquez, E. Also, C. Iemmi, J. Campos and M.J.Yzuel, "Influence of the temporal fluctuations phenomena on the ECB LCoS performance," Proc. SPIE 7442, 74420G-1 (2009).

[16]A. Lizana, I. Moreno, A. Márquez, C. Iemmi, E. Fernández, J. Campos and M. J. Yzuel, "Time fluctuations of the phase modulation in a liquid crystal on silicon display: characterization and effects in diffractive optics," Opt. Express 16, 16711-16722 (2008).

[17]A. Lizana, A. Márquez, L. Lobato, Y. Rodange, I. Moreno, C. Iemmi, and J. Campos, "The minimum Euclidean distance principle applied to improve the modulation diffraction efficiency in digitally controlled spatial light modulators," Opt. Express 18, 10581-10593 (2010).

[18]A. Márquez, C. Cazorla, M. J. Yzuel and J. Campos, "Characterization of the retardance of a wave plate to increase the robustness of amplitude-only and phase-only modulations of a liquid crystal display," J. Mod. Opt. 52, 633-650 (2005).

[19]A. Márquez, F. J. Martínez, S. Gallego, M. Ortuño, J. Francés, A. Beléndez, and I. Pascual, "Classical polarimetric method revisited to analyse the modulation capabilities of parallel aligned liquid crystal on silicon displays," Proc. SPIE 8498, 84980L (2012). 
[20]C. Ramirez, B. Karakus, A. Lizana, and J. Campos, "Polarimetric method for liquid crystal displays characterization in presence of phase fluctuations," Opt. Express 21, 3182-3192 (2013).

[21]A. Hermerschmidt, S. Osten, S. Krüger and Thomas Blümel, "Wave front generation using a phase-only modulating liquid-crystalbased micro-display with HDTV resolution," Proc. SPIE 6584, 65840E (2007).

[22]J. R. Moore, N. Collings, W. A. Crossland, A. B. Davey, M. Evans, A. M. Jeziorska, M. Komarčević, R. J. Parker, T. D. Wilkinson and H. Xu, "The silicon backplane design for an LCOS polarization-insensitive phase hologram SLM,” IEEE Photon. Techol. Lett. 20, 60-62 (2008). 